Performance and application of heavy ion nuclear microbeam facility at the Nuclear Physics Institute in Řež, Czech Republic

Oleksandr Romanenko, Vladimir Havranek, Anna Mackova, Marie Davidkova, Mariapompea Cutroneo, Alexander G. Ponomarev, Gyula Nagy, and James Stammers

Citation: Review of Scientific Instruments 90, 013701 (2019); doi: 10.1063/1.5070121

View online: https://doi.org/10.1063/1.5070121

View Table of Contents: http://aip.scitation.org/toc/rsi/90/1

Published by the American Institute of Physics

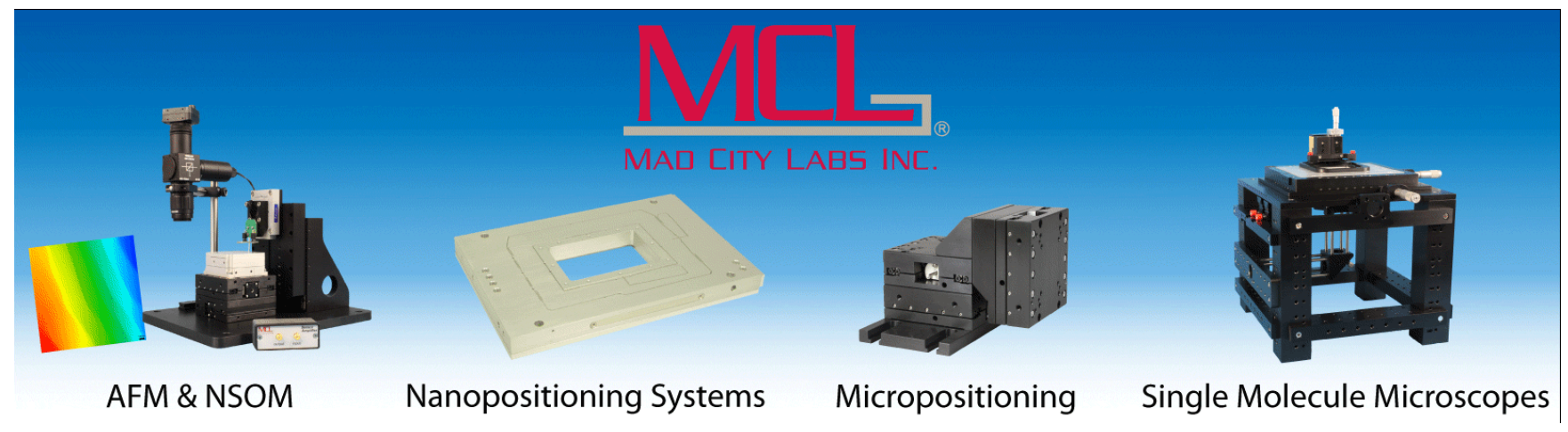




\title{
Performance and application of heavy ion nuclear microbeam facility at the Nuclear Physics Institute in Řež, Czech Republic
}

Cite as: Rev. Sci. Instrum. 90, 013701 (2019); doi: 10.1063/1.5070121

Submitted: 19 October 2018 - Accepted: 12 December 2018 •

Published Online: 7 January 2019

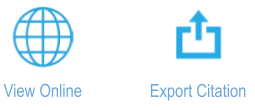

Vladimir Havranek, ' Anna Mackova, ${ }^{1,2}$ Marie Davidkova,

Oleksandr Romanenko,

\section{Mariapompea Cutroneo, ${ }^{7}$ (i) Alexander G. Ponomarev, ${ }^{3}$ Gyula Nagy, ${ }^{4}$ and James Stammers}

\author{
AFFILIATIONS \\ ${ }^{1}$ Nuclear Physics Institute of the Czech Academy of Sciences, Řež 250 68, Czech Republic \\ ${ }^{2}$ Department of Physics, Faculty of Science, J. E. Purkinje University, Ceske Mladeze 8, Usti nad Labem 400 96, Czech Republic \\ ${ }^{3}$ Institute of Applied Physics, National Academy of Sciences of Ukraine, Sumy 40030, Ukraine \\ ${ }^{4}$ Institute for Nuclear Research, Hungarian Academy of Sciences (MTA Atomki), Bem tér 18/c, Debrecen H-4026, Hungary
}

a) Author to whom correspondence should be addressed: romanenko@ujf.cas.cz

\begin{abstract}
The Tandetron Laboratory of the Nuclear Physics Institute of the Czech Academy of Sciences is equipped with five beam lines associated with a $3 \mathrm{MV}$ tandem electrostatic accelerator model $4130 \mathrm{MC}$ from High Voltage Engineering Europa B.V. This accelerator is coupled with two duoplasmatron sources and a single sputter ion source and provides ions from hydrogen to gold. One of these lines is a nuclear microbeam facility, utilizing ion beams of micro- and sub-micro sizes for materials research by use of particle induced x-ray emission spectroscopy, particle induced gamma emission, Rutherford back-scattering spectroscopy, and scanning transmission ion microscopy methods as well as for ion beam writing. The major advantage of the presented microprobe is a possibility of 3D structure creation not only in polymer materials using light ions but also in other materials such as glass, ceramics, etc. by use of heavy ions. The focusing system allows focusing of charged particles with a maximum rigidity of 11 $\mathrm{MeV}$ amu $/ \mathrm{q}^{2}$. The usual resolution in high and low current modes is $2 \times 3 \mu \mathrm{m}^{2}$ for a $100 \mathrm{pA}$ and $0.3 \times 0.5 \mu \mathrm{m}^{2}$ for the 2000 ions /s of $2 \mathrm{MeV}$ protons, respectively. A detailed facility description is given in the paper. The applications of focused beams of heavy ions as well as examples of light ions utilizing are also presented in the article.
\end{abstract}

Published under license by AIP Publishing. https://doi.org/1 0.1063/1.5070121

\section{INTRODUCTION}

Energetic ion beams are widely used to analyze the composition and structure of the materials. Environmental samples, biological objects, geological materials, electronic devices, photonics materials, and different types of solids can be investigated by ion analytical methods ${ }^{1-5}$ which have a number of unique features and cannot be replaced by other alternative approaches for qualitative and quantitative material analysis. Particle Induced X-ray Emission spectroscopy (PIXE) and Rutherford Back-Scattering spectroscopy (RBS) are the most commonly used methods in ion beam analysis that allow researching of trace elements in materials as a result of outstanding sensitivity and elements distribution in depth, respectively. Other nuclear methods supplement the major mentioned methods and help to investigate hydrogen concentration in materials by Elastic Recoil detection Analysis (ERDA), 6,7 determine defects in single-crystalline materials by RBS-channeling, ${ }^{8}$ obtain maps of structures of thin materials by Scanning Transmission Ion Microscopy (STIM), ${ }^{9}$ etc. The obtained experimental data are further used to characterize the composition of structures in material research, radiation studies, dosimetry, study of nuclear reaction cross section, etc.

Ion beams are also used for modification of materials. ${ }^{10-14}$ Mechanical, electrical, magnetic, optical, chemical, and biocompatible properties can be changed by ion irradiation. Due 
to well-controlled parameters of irradiation, the properties of the materials can be varied very accurately. This helps in researching novel materials with specific properties.

The nuclear microbeam facility (microprobe) is a unique facility, which enables the use of ion beams of micro- and nanometer size at high enough currents, in the investigation of materials by standard nuclear methods. It permits use of the same nuclear methods as for macrobeam measurements but has the added advantage of building a map of the element distribution of the materials as well as reconstruction of the 3D structure of the sample (PIXE and STIM tomography $\left.{ }^{15,16}\right)$. In addition, STIM, which constructs a map of density and thickness for a given sample, is used only on the microprobe.

The microbeam can be used to generate microstructures with the ion beam writing process, wherein the ion beam, on radiation-sensitive materials, produces microstructures that have significant optical and electrical properties. ${ }^{17-19}$ Samples that cannot be placed in a vacuum can also be examined in the air using the external ion beam, which is an extension of the microprobe such that the ion beam is drawn into the atmosphere through the thin window at the end of the microbeam chamber and the external detectors record the produced Xray radiation in the air. ${ }^{20,21}$ Ion beam writing and machining is a rising scientific branch due to the application for microand nano-structure synthesis and modification of 2D materials such as polymers and semiconductors. At current, the use of focused heavy ion beams proves very promising for $\mathrm{MeV}$ Secondary Ion Mass Spectrometry (SIMS) and versatile material microstructuring applications. ${ }^{22,23}$ Thus, improvement of the ion microbeam lateral resolution, testing of the focused heavy ion beam, precision beam manipulation, and upgrading of the precise and continuous microbeam current controller are all highly important and the focus of the present article.

The Tandetron accelerator is a part of the recently established Center of Accelerator and Nuclear Analytical Methods (CANAM). The large research infrastructure, CANAM, is a center for the investigation of tasks in a wide range of scientific disciplines using beams of accelerated ions and neutrons. CANAM has been incorporated in the "Roadmap of Large Infrastructures for Research, Experimental Development and Innovation of the Czech Republic" since 2010. CANAM includes, with the exception of the Tandetron Laboratory, the isochronous cyclotron U-120M, the IBA C70 cyclotron ARRONAX, and the neutron beam laboratory at research reactor LVR-15 (owned by Research Centre Rez). Nuclear Physics Institute of the Czech Academy of Sciences (NPI CAS) offers access for external users to its experimental facilities of the CANAM infrastructure in open access mode. Access to the facilities in the Laboratory of Cyclotron and Fast Neutron Generators, Laboratory of Tandetron, and Neutron Physics Laboratory is free of charge for national and international academic users, provided that the results from experiments at the facilities of the NPI infrastructure will be disseminated in the public domain. ${ }^{24}$

\section{MICROPROBE AND EXTERNAL BEAM}

\section{A. Microprobe beam line and beam line vacuum system}

In 2005, we replaced an old accelerator (Van de Graaff type) with the new tandem electrostatic accelerator from High Voltage Engineering Europa B.V. (model 4130 MC). It allows acceleration of a wide spectrum of ions from hydrogen to gold and with energies from one hundred $\mathrm{keV}$ up to about $20 \mathrm{MeV}$ and significantly extends experimental possibilities in the field of analyzing and modifying substances with ion beams. The accelerator is coupled with cesium sputter ion source (HVE, model 860A) for production of negative heavy ions and two duoplasmatrons (HVE, model 358). The first is used to produce positive helium particles and equipped with the Li recharging channel setup; the second produces negative hydrogen ions or $\mathrm{NH}$-molecular ions which are subsequently used for producing nitrogen beams. In duoplasmatron, hydrogen is generated from a gas $\mathrm{H}_{2}$ and $\mathrm{NH}$-molecular ions from a gas mixture of $\mathrm{H}_{2}$ $+\mathrm{N}_{2}$. In sputter source, $\mathrm{H}^{-}$and $\mathrm{NC}^{-}$are generated from targets of $\mathrm{TiH}$ and $\mathrm{BN}$-with-graphite, respectively. The beam brightness for hydrogen obtained with the duoplasmatron source is one order higher than that for the sputter source. The same situation is with nitrogen. Thus, for the microbeam application, we prefer the duoplasmatron source as it provides higher beam current for high current microbeam application with the same resolution. In special case, when a stable beam with a not so high current is needed, the sputter source for hydrogen and nitrogen beam is used. A measured axial beam brightness for $2 \mathrm{MeV}$ proton beam provided by duoplasmatron has a value of about $3 \mathrm{pA} /\left(\mu \mathrm{m}^{2} \operatorname{mrad}^{2} \mathrm{MeV}\right)$.

The foundation in the experimental hall is made from a concrete block with an upper isolation layer. A schematic arrangement of the analytical end stations based on the tandem electrostatic accelerator is presented on Fig. 1. A switching magnet has exit ports at $+30^{\circ},+10^{\circ}, 0^{\circ},-10^{\circ}$, and $-30^{\circ}$. The 0 th channel was equipped with the additional magnet recently and is devoted to ion implantation. A condenser system is located before the switching magnet. It is constructed from three electrostatic quadrupole lenses and enables matching of a beam emittance with a system acceptance. A complex system for remote controlling and monitoring of valves, Faraday cups, correction coils, and video cameras was developed in our laboratory and implemented for the routine use of Tandetron operators. This gives them full control over all vacuum parts of the ion beam lines as well as monitoring, focusing, and collimating systems of the ion beam through all lines including the end station vacuum chambers. This system was installed at the accelerator control desk; therefore, operators can drive the beam through the whole line and see the beam into the chamber with the samples. This helps to adjust parameters of the ion source, accelerator, deflection, and condenser systems in order to obtain the best beam quality for the experiment. At the present time, all analytical lines work in routine regime and cover almost all methods of ion beam analysis. We provide the broad portfolio of analytical methods based on elastic ion interaction with solids [RBS, ERDA, 


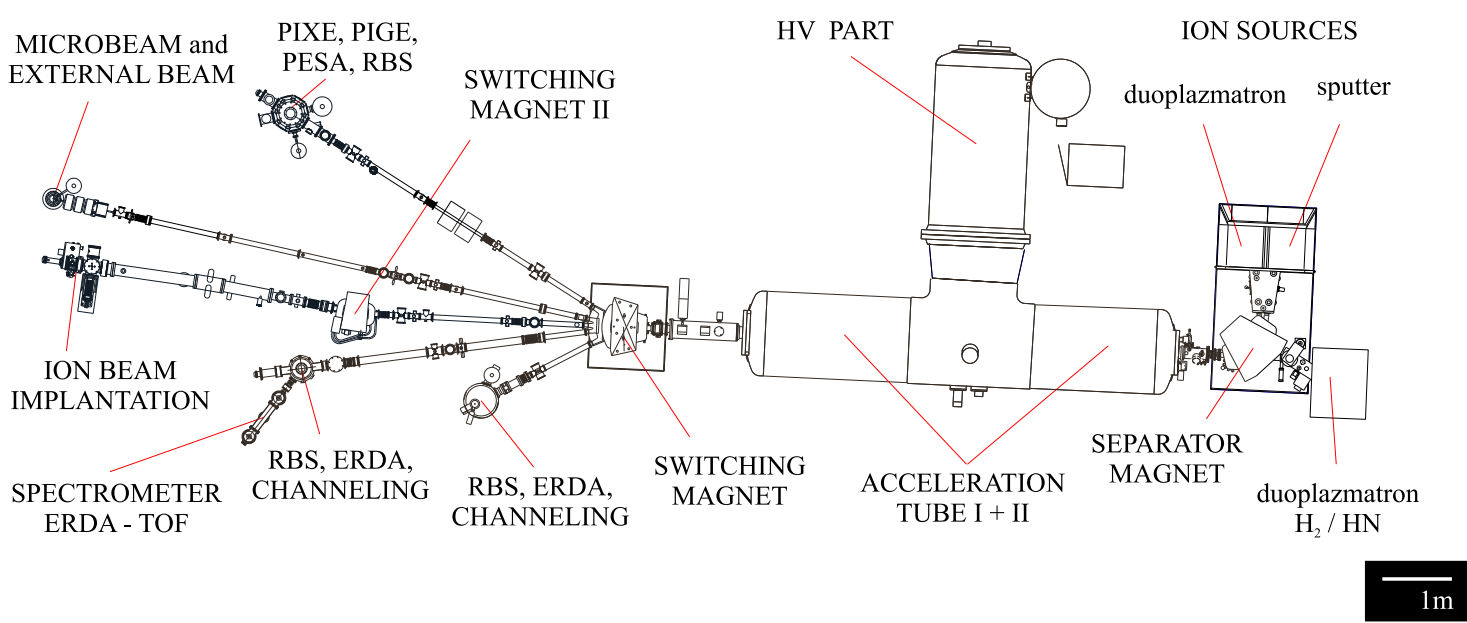

FIG. 1. Schematic diagram of the beam lines attached at Tandetron and the associated instrumentation facilities at NPI CAS

ERDA equipped with Time of Flight (TOF) spectrometer] for elemental precise depth profiling, RBS-channeling in single crystalline materials for structure studies, moreover nuclear reaction analysis - PIGE (particle induced gamma emission) for light elements analysis, PIXE, PESA (Proton Elastics Scattering Analysis), STIM for the detailed elemental composition studies, 2D mapping of elements, trace element analysis, using microbeam microstructure visualization, ion beam implantation for nanostructuring, doping, and tailoring of material electric, optical, mechanical, and other properties.

The microprobe is positioned at a $10^{\circ}$ angle to the switching magnet. Figure 2 presents a scheme of the microprobe and a photo of the microbeam end-station at NPI CAS. In order to minimize vibrations, the object slits and the end-station are supported by separate systems, which are made from the concrete block. Bellows are used for making vibration isolation between the parts. Two correction coils (D1 and D2), rotated by $90^{\circ}$ relative to each other, allow to change ion beam trajectory in two perpendicular directions. Preliminary slits limit the beam current impinging on the object slits and protect them from additional heat load and thereby prevent slit jaws damage. Two Faraday cups (F1 and F2) (NEC, model FC50) are located on an automotive moving stage and allow the operator to control the beam current before and after object collimator. From this the optimum size of the preliminary slits, the part of the beam cut by the object collimator, and the parameters of the condenser system can be determined. The blanking system (DF) is represented by a fast electrostatic deflector

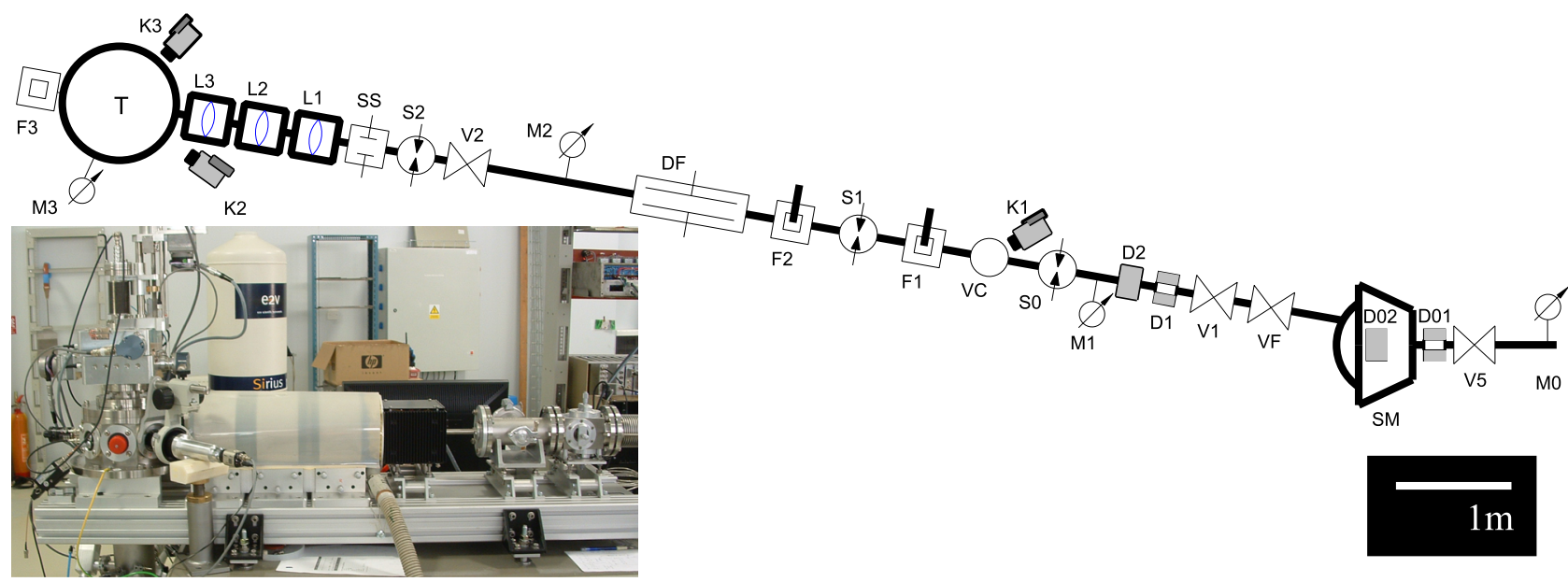

FIG. 2. Scheme of the nuclear microbeam channel. Shorthand notation: SM—switching magnet; V—valve; VF-fast-acting automatic valve; D—correction coil; M-vacuum meter; S0 - preliminary slits; S1—object collimator; S2-angular collimator; VC-viewing chamber; K-videocamera; F-Faraday cup; DF-fast deflector; SS-scanning system; L-magnetic quadrupole lens; and T-target chamber. 
constructed from two parallel plates of $1 \mathrm{~m}$ length. It is controlled by the logic system and has $1 \mu$ s rise time of a voltage on the plates to $100 \mathrm{~V}$.

The valve V2 divides the vacuum system of the channel into two parts. This allows for vacuum pumping of two parts separately. The vacuum of the first part (according to the beam direction) is generated using pre-pump and turbo molecular pump $(60 \mathrm{l} / \mathrm{s})$ pairs, located halfway along the line. The vacuum of the target chamber is provided by the oil-free pre-pump and special turbo molecular pump (Pfeiffer Vacuum model HiPace 300plus, 260 l/s), which does not add vibration to the chamber and allows a fast recovery of vacuum after sample changes. The vacuum in the target chamber during a measurement is about $10^{-6}$ Torr. In the necessary case of opening the chamber for sample changes, it is filled with $\mathrm{N}_{2}$ gas, which is better than air in terms of obtaining a vacuum. The vacuum of $10^{-5}$ Torr can be achieved within 10 min which allows the dividing valve to be opened and a new measurement to begin.

A fast-acting automatic valve (VF) protects the accelerator in the case of a rupture of the window in the chamber exit port during an external ion beam irradiation. The distance between the valve and the beam exit window is $8 \mathrm{~m}$. If the pressure inside target chamber exceeds $1.5 \times 10^{-5}$ Torr, the valve closes within $10 \mathrm{~ms}$ and separates off accelerator part from the microprobe before air reach the valve.

\section{B. Microprobe end station}

The target chamber is based on the OM70 design (Oxford Microbeams Ltd.), with UHV Design Ltd. 3-axis Multibase positioning stage [range $\mathrm{x}= \pm 10 \mathrm{~mm}, \mathrm{y}= \pm 50 \mathrm{~mm}$, and $\mathrm{z}$ (corresponds to ion beam axis) $= \pm 10 \mathrm{~mm}]$. Micromanipulator equipped with stepper motors provides $2 \mu \mathrm{m}$ resolution. Figure 3 shows the inner view of the target chamber. In the

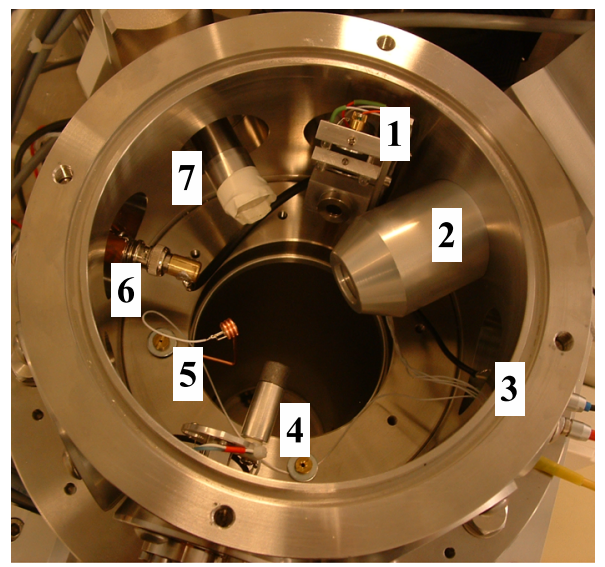

FIG. 3. Inside view of the target chamber: (1)-ion beam entrance, RBS detector, optical microscope without magnification and LED, UV, IR lamps; (2)optical microscope with magnification $\times 160$; (3) -inlet for illumination cables; (4) Faraday cup and STIM detector; (5)_-socket for a glow lamp without a glass bulb; (6)_inlet for RBS detector cable; and (7)_-PIXE detector. vertical chamber walls, there are 8 windows located at $45^{\circ}$ and sealed with flanges. There is a PIPS detector (position 1 Fig. 3) used for RBS measurement at the ion beam entrance. An optical microscope (position 2 Fig. 3) bases on the CCD camera (WATEC model WAT-250D) with the optical system and provides a magnification of $\times 160$. This is quite enough to observe not only the samples but also the fluorescent pattern of the spot shape on the quartz target during beam focusing procedure.

The additional camera is located above the PIPS detector and has no optical magnification and thus provides a general view of the inside of the chamber. LED, UV, and IR lamps are located in the 1 position and allow us to illuminate the chamber interior with different light wavelengths. A glow lamp without a glass bulb in position 5 emits electrons when it is turned on and thus allows the accumulated (on the sample) positive charge to escape. This is very important when irradiating insulators. Otherwise, the accumulated positive charge can deflect the incident ion beam and spoil the desired structure, for example, in glasses, as we observed in our experiment. ${ }^{18}$ An ultra-sensitive current monitor system (Oxford Microbeams Ltd. model OM35e) includes a Faraday cup (position 4 Fig. 3) and allows measurement of the ion beam current in the range from $0.15 \mathrm{pA}$ to $1 \mathrm{nA}$. The detector for on-axis STIM measurements is located on the same stage as the Faraday cup (position 4 Fig. 3). The detector for PIXE measurements is located at $45^{\circ}$ to the beam (position 7 Fig. 3). There are two wires leadingout, situated opposite each other. The first of them is used for the RBS detector (position 6 Fig. 3) while the second-for lighting (position 3 Fig. 3).

The end station is currently equipped with the PIXE, RBS, and STIM setups, as shown in Fig. 3. X-rays are detected by e2V Scientific Instruments (now RaySpec Ltd.), model Sirius 80. This model is optimized for low-energy X-rays detection. It is an EDX $\mathrm{Si}(\mathrm{Li})$ semiconductor detector with $80 \mathrm{~mm}^{2}$ active area, $4 \mathrm{~mm}$ crystal thickness, and a $12 \mu \mathrm{m}$ Be window, having a resolution of $150 \mathrm{eV}$ with $1 \mu \mathrm{s}$ shaping time at the $\mathrm{Mn} \mathrm{K}_{\mathrm{a}}$-line. The detector has a fluoroplastic nozzle, used for absorption of low-energy X-ray with high load rate. The thickness of the fluoroplast is chosen depending on the composition of the sample. The detector is mounted on the stage, and the distance to the sample can be changed manually. The angle between chamber entrance for the beam and detector is $45^{\circ}$.

The passivated implanted planar silicon (PIPS) detector (manufactured by Canberra) is used for RBS measurements. This annular $\mathrm{Au}(\mathrm{Si})$ surface barrier detector has an active area of $50 \mathrm{~mm}^{2}$ and is located at $60 \mathrm{~mm}$ from the sample stage. At present time, it has a resolution of $15 \mathrm{keV}$. A generated signal proceeds to a charge sensitive preamplifier (Canberra, model 2003BT), to a spectroscopy amplifier (Canberra, model 2024), and then to a signal analyzer (OM, model 1000e). The detector is located at $20^{\circ}$ to the beam (in the RBS geometry, it means a scattering angle $\theta=160^{\circ}$ ).

A windowless $\mathrm{Si}$-PIN photodiode (made by Hamamatsu, model S3590) is used for detection of transmitted beam 
particles through the sample. This cheap detector has a resolution comparable to the PIPS detector for detecting charged particles with an energy of several MeV. ${ }^{25}$ It has a $0.3 \mathrm{~mm}$ thickness and $1 \mathrm{~cm}^{2}$ active area. The distance between the sample stage and the detector is $10 \mathrm{~cm}$. It is combined with a preamplifier (Canberra, model 2003BT) and an amplifier (Canberra, model 2026).

The signals from all detectors are connected to the OM1000e interface unit, where they are converted to digital signals. The OM-DAQ system consists of OM1000e module, PC under MS Windows XP, and a special software. It collects data from detectors simultaneously and sorts them into spectra. In addition, it controls the scanning system and allows the elemental maps to be built up to $512 \times 512$ pixels. ${ }^{26}$

\section{Microprobe forming and scanning system}

As Oxford Microbeams OM2000 end-station is used in our microprobe channel, the focusing system is represented by a triplet of magnetic quadruplet lenses (model OM50). This type of the focusing system uses converging-divergingconverging configuration for the $\mathrm{x}$-plane. First and second lenses along the beam path are connected with one power supplier, while the last lens-with second one (model OM52e). Lenses are housed in air duct for cooling. It permits a decreased beam shift at the target as a result of the thermal shift of lenses. Each lens has a thermometer. In addition, one thermometer measures the air temperature in the duct (outgoing air), and the second one-the ambient temperature (incoming air). The lens temperature controlling system was developed in our laboratory and enables visualize temperature change with time and records it. It helps to determine the time when the lens temperatures reach an equilibrium state. If the experiment starts after the equilibrium point and the ambient temperature is stable, the beam shifting on the sample will not occur as a result of the thermal shift of lenses. A measured value of the beam shift at the target by lens temperature change is $5 \mu \mathrm{m}$ per $1{ }^{\circ} \mathrm{C}$. Figure 4 represents the record that was created during micromachining experiments with $10.5 \mathrm{MeV} \mathrm{N}^{4+}$ with the current of the lenses 86 and 89 A (note: this is the uploaded data that were recorded earlier, and thus the temperature values are indicated as "OFF"). As can be seen, the time of the lenses temperature saturation is about $1.5 \mathrm{~h}$. Object and angular collimators are constructed by Oxford Microbeams LTD. and can set the window dimension with accuracy 1 and $10 \mu \mathrm{m}$, respectively.

For calculations of the parameters of the probe forming system, such as demagnifications, chromatic, and spherical aberration, PROBForm code ${ }^{27}$ based on the matrizant method $^{28}$ was used. The dimensions of the microprobe and the results of calculation are given in Table I. The maximum mass energy product is limited by lens power supplier at level $11 \mathrm{MeV}$ amu/q $\mathrm{q}^{2}$. In this case, the first and second lenses have nearly $93 \mathrm{~A}$, and the third-96 $\mathrm{A}$, and the maximum pole tips fields lay below 0.42 and $0.45 \mathrm{~T}$, respectively.

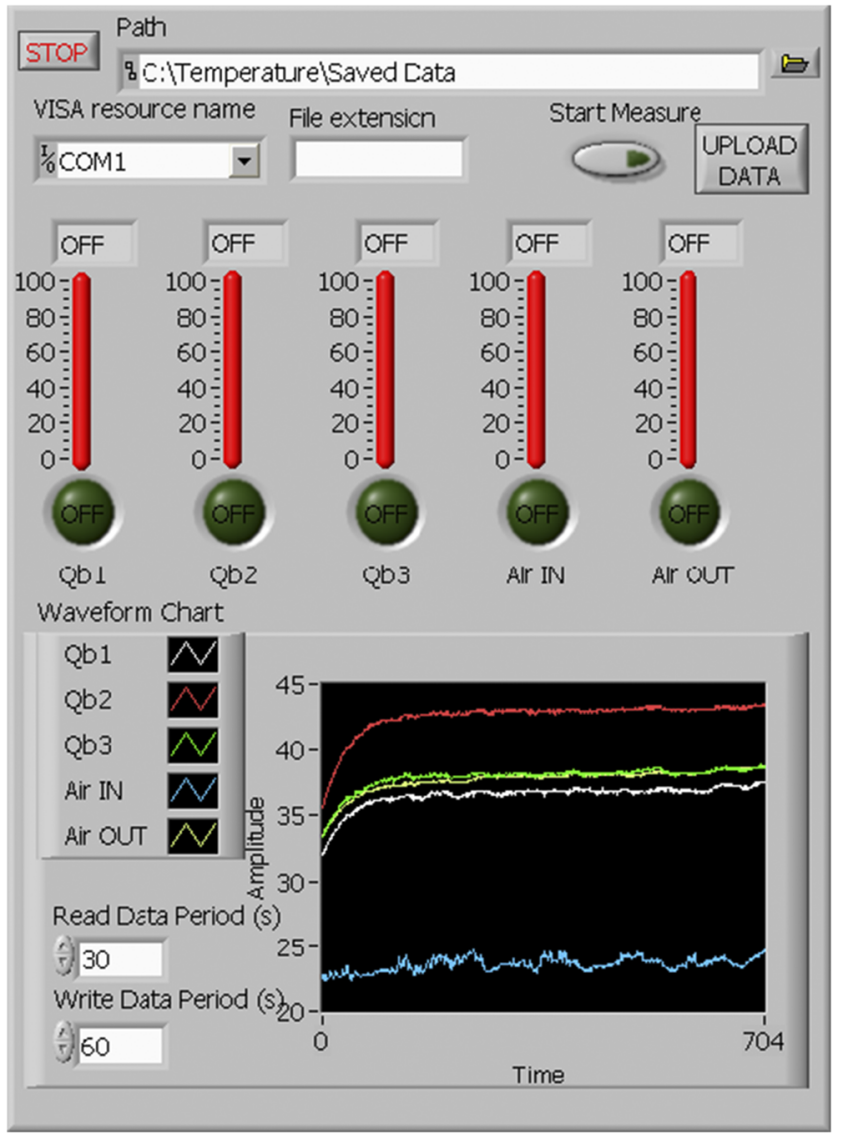

FIG. 4. Lens temperature monitoring (shown record of the micromachining experiment with $10.5 \mathrm{MeV} \mathrm{N}^{4+}$ ).

The scanning system (model OM25) is located before the focusing system and bases on ferromagnetic coils. The maximum raster and resolution are $2 \times 2 \mathrm{~mm}^{2}$ and 512 points,

TABLE I. Ion optic parameters for the probe forming system of the presented microprobe.

System length (distance between object and target) ( $\mathrm{mm}$ )

Object distance (distance between object

and angular collimator) ( $\mathrm{mm}$ )

Lens lengths $(\mathrm{mm})$

Distance between lenses ( $\mathrm{mm})$

Working distance $(\mathrm{mm})$

Lens aperture radius (mm)

Demagnification Dx, Dy

Chromatic aberrations $[(\mu \mathrm{m} / \mathrm{mrad}) / \%]$

$\mathrm{C}_{\mathrm{px}}$

Intrinsic spherical aberration $\left(\mu \mathrm{m} / \operatorname{mrad}^{3}\right)$

$\left\langle x / \theta^{3}\right\rangle$

$\left\langle x / \theta \varphi^{2}\right\rangle$

$\left\langle y / \varphi^{3}\right\rangle$

$\left\langle y / \theta^{2} \varphi\right\rangle$

5046

100

50

170

7.5

$53.2 ;-16.5$

$-236$

585

101

158

$-580$

$-508$ 
respectively. For controlling the scanning system, a twocoordinate power supply (model OM40e) is used and synchronized with the data acquisition system by OM1010e module. The stand time at one point during scanning procedure varies from $1 \mu \mathrm{s}$ to $10 \mathrm{~s}$. It is also possible to perform scan in chargecollection regime. In this case, the accumulated charge at one point can be set in a range of $1 \mathrm{fC}-1 \mathrm{nC}$. In the case that the size of the area provided by the scanning system is not enough for sample measurement or modification, the sample positioning system permits an increase in the area of interest up to $2 \times 10 \mathrm{~cm}$.

\section{PERFORMANCE OF THE MICROPROBE}

Usually the microprobe is used in two opposite regime: (1) high current regime (above $100 \mathrm{pA}$ )-for performing solid sample investigation using such popular microbeam analysis methods as $\mu$-PIXE and $\mu$-RBS and for micromachining; (2) low current regime (below $10 \mathrm{fA}$ )-for STIM analysis and for biological samples irradiation. As the main aim of the microprobe development is to achieve the minimum ion beam size at the target plain with sufficient current for analysis, it is obvious that the beam current is varied by collimators, not by decreasing beam current at the entrance of the microprobe channel. This results in a different resolution dependent on the regime. In ideal circumstances, the beam size on the target is determined by the object window (in case of keeping angular divergence of the beam by collimating slits) because the demagnification and aberration coefficients are constant for the selected probe forming system. However, in practice, the beam size does not decrease proportionally with decreasing the object window due to, for instance, mechanical vibration, ion scattering on the slits, or magnetic stray fields. Therefore, it is useful to indicate the resolution for each regime in order to compare performance of different microbeam facilities.

Performance of the system was tested by the $\mu$-PIXE method for a standard copper mesh manufactured by Gilder Grids Ltd. (1000 lines/in). Two orthogonal line scans were used to obtain the yield profiles of the interaction products of the ion beam with the material [Fig. 5(a)]. The standard OMDAQ software is used to estimate the beam size at FWHM, but it uses simplified method and therefore gives rough values [our observations showed that this method always underestimates the beam size (shows a better resolution, than in reality)]. That is why we used values provided by OMDAQ package only for finding the minimum beam size during focusing procedure, but for evaluation FWHM, we selected more precise method. The beam's FWHM was extracted by fitting a theoretical profile with assumption of a Gaussian beam profile. A fitting procedure was performed by use of an error-function in a Mathematica software environment. The minimum beam spot size about $1.9 \mu \mathrm{m}$ and $3 \mu \mathrm{m}$ in the $\mathrm{X}$ and $\mathrm{Y}$ directions, respectively, was obtained with a $2 \mathrm{MeV}$ proton beam with a current of $100 \mathrm{pA}$ [Fig. 5(b)].

For the beam size determination in low current regime, the STIM method was used. For this measurement, the same copper mesh as for PIXE measurement with 1000 lines/in was used. The resolution of $0.3 \times 0.5 \mu \mathrm{m}^{2}$ at a beam current of 2000 ions/s was determined (Fig. 6).
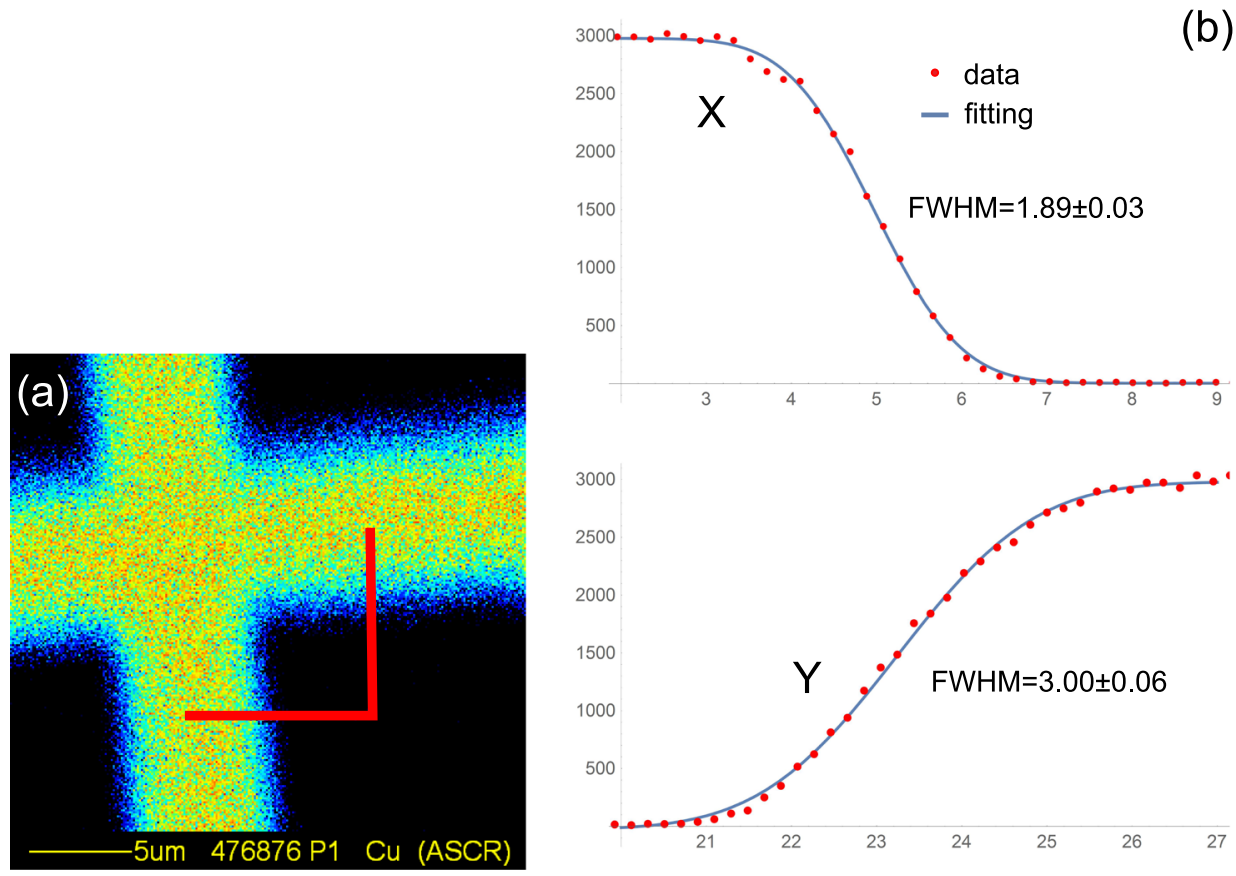

FIG. 5. Beam size evaluation: (a) PIXE image of the copper mesh. (b) Profiles of $X$-ray yield which correspond to two orthogonal scan lines obtained by $2 \mathrm{MeV}$ proton beam with $100 \mathrm{pA}$ current. 

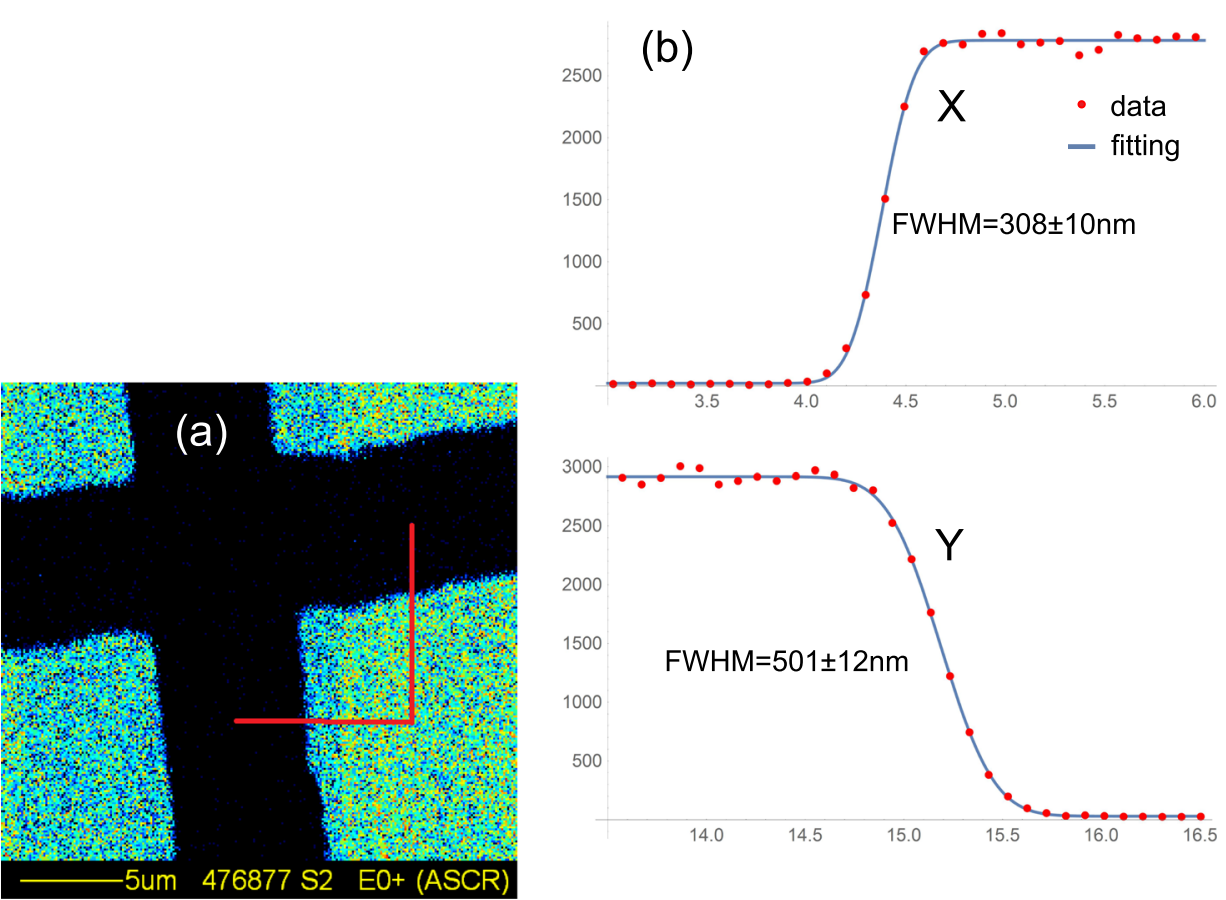

FIG. 6. Beam size evaluation: (a) STIM image of the copper mesh. (b) Profiles of X-ray yield which correspond to two orthogonal scan lines obtained by $2 \mathrm{MeV}$ proton beam with the current of 2000 ions/s.

\section{MICROBEAM MACHINING-REALIZATION}

Microbeam machining makes high demands for the scanning system in comparison with other ion beam method. In case of obtaining maps of elements distribution or material thickness by $\mu$-PIXE or STIM methods, respectively, a simple scan of the selected square region without interruption between each pair of points is enough. For micromachining, the scan shape is usually complicated. It is possible to create an intricate pattern using the standard method of scan with the square region by interrupting the beam. For doing this, a fast electrostatic deflector is needed. The description of our blanking system is given above in Sec. II.

For realization of microbeam machining, special software was developed at our laboratory (Fig. 7). LabView was selected as the most appropriate programming environment for such type of tasks. The program needs a bitmap file with a pattern for irradiation and imposes a limit on resolution of an image of $4 \mathrm{k} \times 4 \mathrm{k}$ pixels. After the user sets up the dose, scan size, measured beam current, blanking time, and number of scan cycles, the program will calculate the step size (marked in program as "point size"), time spent in one pixel, total scan time, and other parameters which are depicted in our microbeam machining software (Fig. 7). The step size is calculated as the scan size divided by resolution of image. For example, if the scan size is $50 \mu \mathrm{m} \times 50 \mu \mathrm{m}$ large and image resolution is at most achievable 512 pixels $\times 512$ pixels, then a scanning step size of the beam is $0.1 \mu \mathrm{m}$. The pattern on Fig. 7 does not have an application but is highly suitable for testing the accuracy of lithography.
The possibility to perform irradiation in several scan cycles is useful when beam current is unstable. In this case, the time of single scan is inversely proportional to the number of cycles, but the total time is the same. The program generates code for the processor of the terminal block BNC-2110 (National Instrument), which executes it and generates voltage in range $0 \ldots+10 \mathrm{~V}$ and logical signal for scan and blanking systems, respectively. The program performs raster scan and moves the beam from the top-left to the bottom-right corner, going from top to bottom only. Generated voltage from terminal block BNC-2110 proceeds directly to the two-coordinate power supply (model OM40e), bypassing OM1010e module, which is controlled by OMDAQ software.

\section{MICROBEAM MACHINING-APPLICATION}

Different types of ions are involved in ion microbeam modification of materials, but light ions are the most widely spread. In the case of protons, the method has its own established name and abbreviation-proton beam writing (PBW).

One of the examples of using light ions is to modify the electron structure of graphene oxide, which is a structural modification of graphene. Graphene oxide is a modification of the $2 \mathrm{D}$ graphene structure where there are additionally oxygen atoms that change the electron structure, so graphene oxide is insulator, unlike the conductive structure of graphene. By modifying the electron structure of the graphene oxide, it is possible to change the energy difference between the valence and the conduction electron band to achieve the 


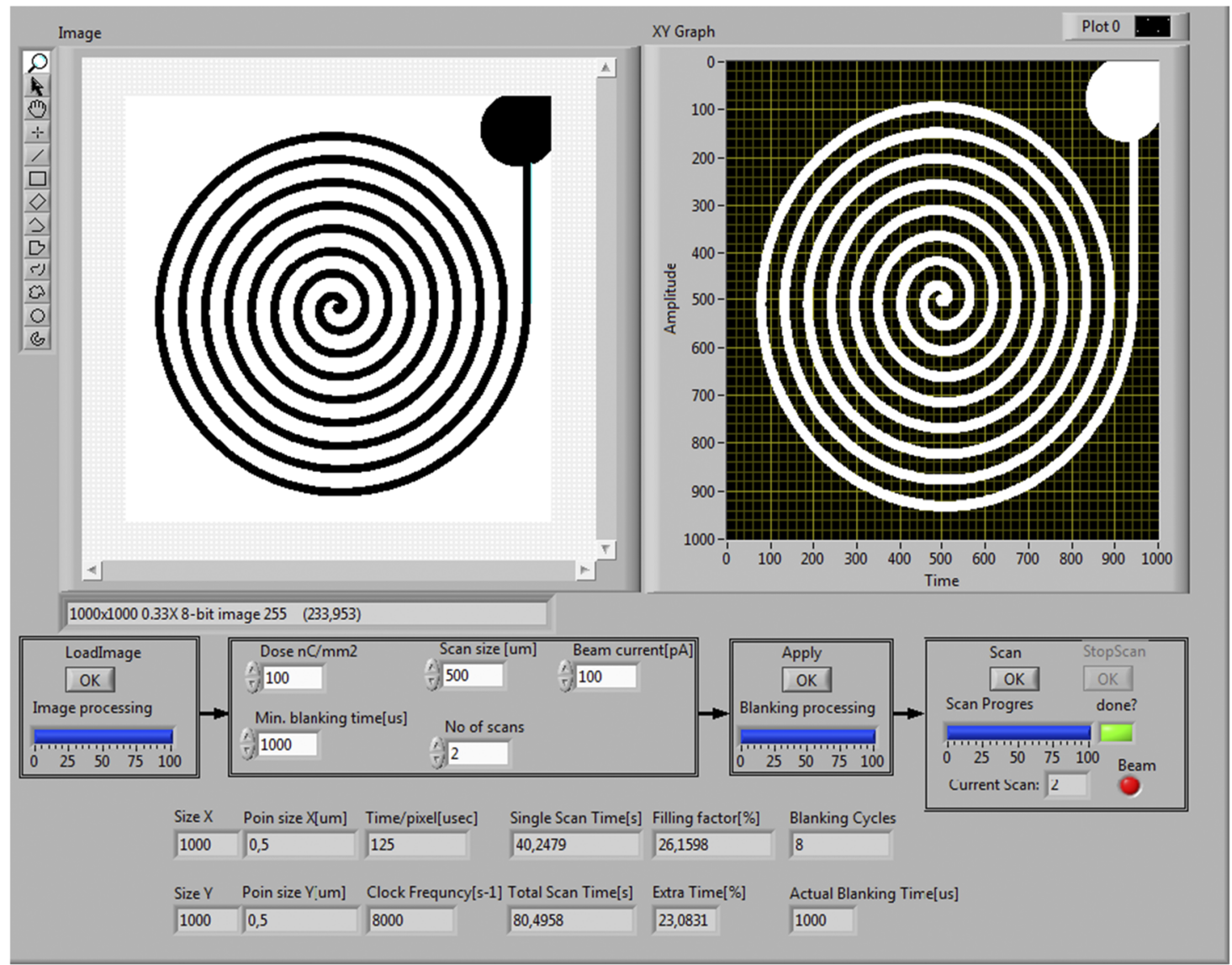

FIG. 7. The interface of the program for microbeam machining.

modification of the isolator to the semiconductor or the conductor. ${ }^{29}$ For investigating the possibility of creating a conductive track in graphene oxide, we scanned a line with a focused $\mathrm{He}^{+}$ion beam with $1.2 \mathrm{MeV}$ energy. The beam was focused into the defined dimensions $20 \times 40 \mu \mathrm{m}^{2}$ with the beam current of 260 pA. Figure 8 represents an optical

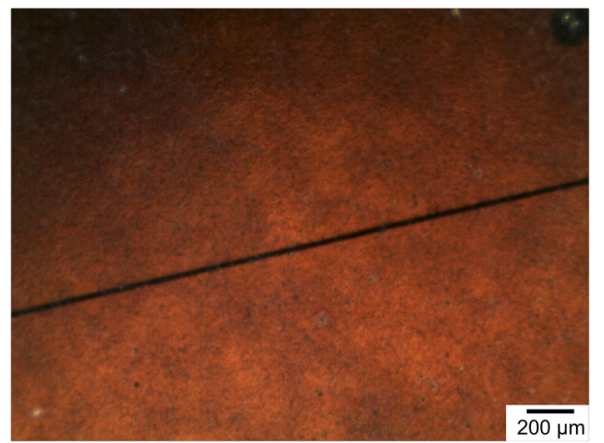

FIG. 8. Graphene oxide foil modified by a $1.2 \mathrm{MeV} \mathrm{He}^{+}$ion beam. The line width is about $20 \mu \mathrm{m}$. image of the created microstructure. The ion beam micromachining in graphene oxide was realized as well as the electronic structure modification. ${ }^{30}$ This shows that ion beam writing is a promising tool for creation of semiconductor or conductive microstructures in the non-conductive graphene oxide.

Heavy ions are also used in our experiments for microbeam machining. For instance, we use them for the creation of micro-optical elements in glasses, crystals, and polymers. The reflective index of optical materials can be easily changed as a result of structural changes in the sample due to ion irradiation, such as swelling/compaction, or crystallization/amorphization. This feature of ion beams is widely used in the fabrication of various optical elements. Direct ion beam writing has advantages over other lithographic techniques in terms of high controllability and reproducibility. A considerable diversity of ion types is available, and the wide range of the ion energy makes it possible to control the depth of the structure with micrometer accuracy.

The possibility of scanning the beam over an area of a few millimeters, using two sets of dipole magnets, makes it 


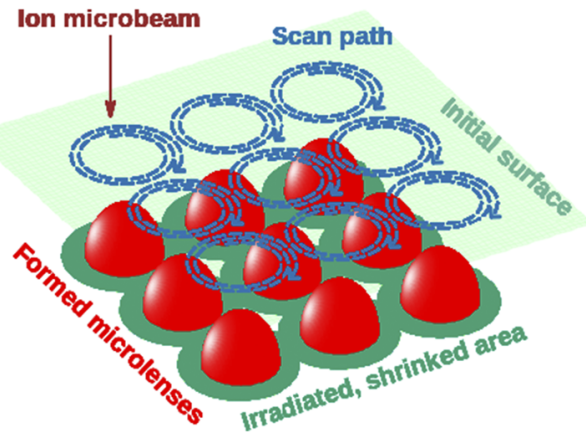

FIG. 9. Schematic drawing of the formation of microlenses in PDMS due to heavy ion microbeam irradiation. Annuli were irradiated along spiral paths, and the material suffers compaction where it is irradiated. The un-irradiated circles within each annulus bend due to the rubbery nature of the material, thus spherical objects form.

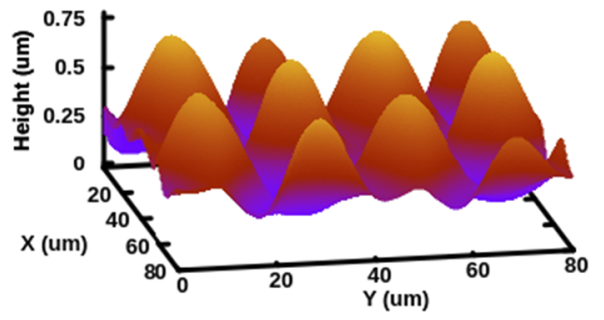

FIG. 10. AFM picture of a microlens array created in PDMS.

possible to write complex structures, such as a micro-lens matrix, while the possibility of scanning the sample stage over several centimeters makes it possible to write simple but extended structures, such as optical waveguides.

In one of the experiments of our laboratory, we realized the micromachining in polydimethylsiloxane (PDMS) using the
10.5 $\mathrm{MeV} \mathrm{N}^{4+}$ microbeam of $2 \times 2 \mu \mathrm{m}^{2}$ size. ${ }^{31}$ First, parallel stripes with various linewidth/periods were irradiated, and the change of the surface morphology was investigated by means of Atomic Force Microscopy (AFM). The structures were created using ion fluence in the range of $7.8 \times 10^{11}-7.8$ $\times 10^{13}$ ions $/ \mathrm{cm}^{2}$. The current of the ion beam was varied in a range 10-200 pA depending on the needed fluence. We found that PDMS suffers compaction due to the heavy ion irradiation that increases asymptotically with the irradiation fluence and saturates after a certain point. Then micro-lens arrays were created using the compaction effect, by irradiating annuli; see schematically in Fig. 9. The focal length of the lenses could be tuned by the degree of compaction, which depends on the diameter of the annuli and the delivered ion fluence. The AFM picture of the created microstructure is shown on Fig. 10.

\section{ANALYSIS OF SAMPLES TREATED BY MICROBEAM MACHINING}

One of the numerous advantages of the ion microbeam facility is based on the possibility of performing analysis by different methods simultaneously. We realize PIXE, PIGE, STIM, and RBS methods, which only represent the methods that can be implemented on the ion microprobe. They complement each other and thus allow enhancing the investigation results of elemental composition and the structure of the materials. Unfortunately, not all methods can be involved at the same time because of detector limitation connected with a maximum count rate. The value of a cross section of the physical processes which take part during interaction of the ions with the target atoms varies for different reactions. This imposes restriction on the beam current used for different methods. As a result, such methods as STIM cannot be used with other methods implicated on our microprobe. Nevertheless, STIM is an excellent method for investigation of the radiation-sensitive materials. Because of the low beam current (typically several thousand ions per second) of light ions, the materials undergo small irradiation dose during analysis. In fact, even such sensitive material as PMMA can be studied
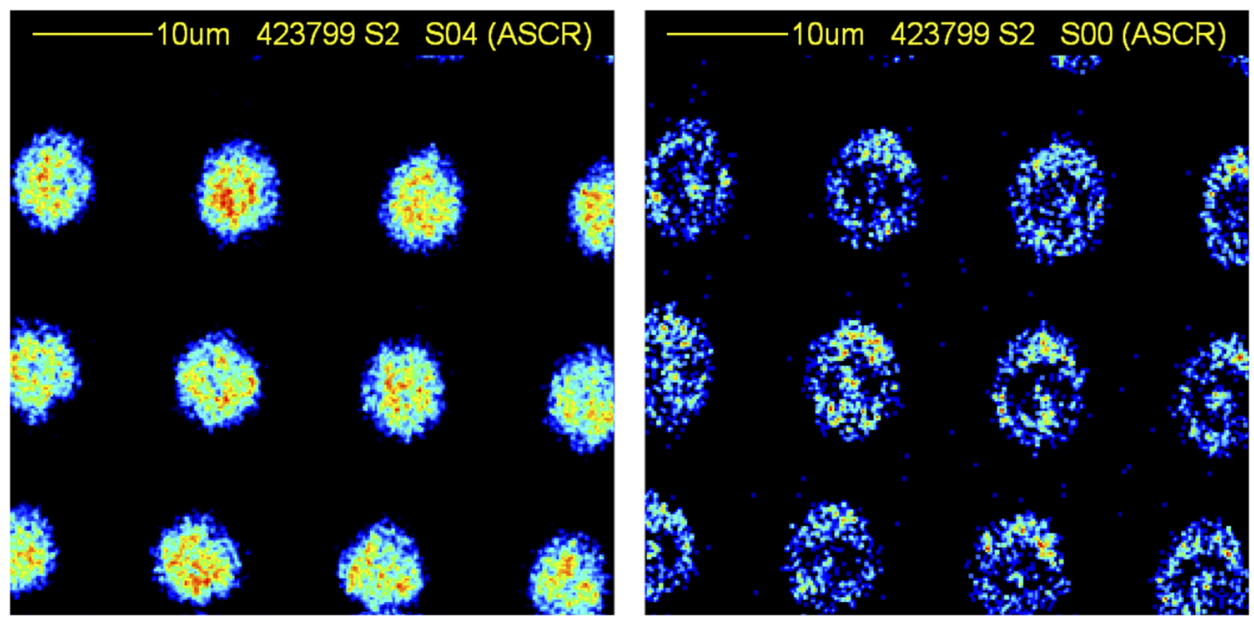

FIG. 11. STIM images of irradiated PMMA obtained by proton beam with energy $2 \mathrm{MeV}$ and current $0.1 \mathrm{fA}$. 
by this method. For example, in our laboratory, we performed analysis of the quality of the bottom and side walls of the produced microstructures in PMMA. The sample PMMA with a thickness of $9 \mu \mathrm{m}$ has been prepared as well as Si substrate spin coated with a $9 \mu \mathrm{m}$ PMMA layer were baked at $180{ }^{\circ} \mathrm{C}$ for $15 \mathrm{~min}$ before irradiation. The proton beam writing experiment was performed at our laboratory using particles of 2 and 2.6 MeV to write structures in PMMA resists. ${ }^{32}$ Performed analysis showed that in spite of $2 \times 2 \mu \mathrm{m}^{2}$ beam size during proton beam writing, the final hole size is about $5 \times 5 \mu \mathrm{m}^{2}$ (Fig. 11), which is explained by post irradiation treatment. In addition, increasing of the hole size with depth means that edges of the holes are not sharply vertical.

\section{EXTERNAL ION BEAM IRRADIATION}

On the backside of the microprobe interaction chamber a flange for external ion irradiation is mounted. The flange has a nozzle with a $200 \mathrm{~nm}$ thick and a $1.5 \times 1.5 \mathrm{~mm}^{2}$ area silicon nitride $\left(\mathrm{Si}_{3} \mathrm{~N}_{4}\right)$ window. The window is strong enough to withstand the pressure drop and allows minimizing both beam shape lateral straggling and energy dissipation due to small thickness. This material is widely used in transmission electron microscopy because of good radiation hardness and mechanical resistance.

The end-stage for external ion beam irradiation is constructed on the metal frame. Samples are fastened on a motorized XY scanning stage manufactured by Standa Ltd. (Fig. 12). The stage has $7.5 \mathrm{~cm}$ travel range in both directions with $2.5 \mu \mathrm{m}$ accuracy. For the Z-direction, we use the manual moving stage. For the beam current measurement, the Si-PIN photodiode is used. It is placed into a metal tube and covered with $1.5 \mu \mathrm{m}$ thickness aluminum foil for the purpose to prevent light influence. The ion current up to $200 \mathrm{kHz}$ can be measured at the shaping time $0.25 \mu \mathrm{s}$. As we used the external ion beam mostly for biological cell irradiation up to now this measurement range of current has covered our requirement. Small current for cell irradiation requires small sizes of collimators as a result the beam size is significantly smaller than the window size. In the case where it is necessary to irradiate a big area and to obtain homogeneous dose distribution over

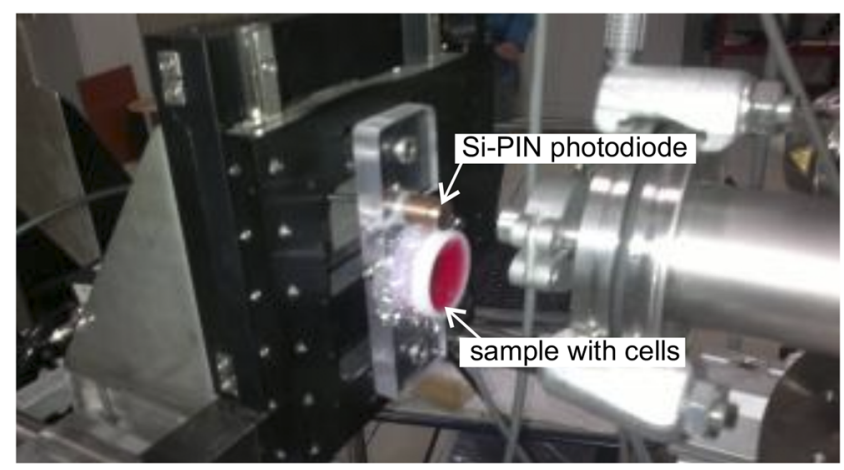

FIG. 12. End-stage for external ion beam irradiation. the whole area, we defocus beam or use scanning procedure so that it fills in the window size.

In order to show the efficiency of external ion beam for cell irradiation on our facility, we represent the results of pilot study where biological efficiency of proton and $\mathrm{He}^{2+}$ particle beams was compared. Human normal neonatal skin fibroblasts were irradiated by $1.1 \mathrm{MeV}$ protons and $2.5 \mathrm{MeV} \mathrm{He} \mathrm{He}^{2+}$ ion beams. Cells were grown on Lumox dishes with a Teflon bottom membrane (Sarstedt) in Dulbecco's Modified Eagle's medium (Sigma-Aldrich) supplemented with 15\% fetal bovine serum (FBS-BIOSERA) and standard antibiotics $[100 \mathrm{U} / \mathrm{ml}$ penicillin and $0.1 \mathrm{mg} / \mathrm{ml}$ streptomycin (Sigma-Aldrich)]. ${ }^{33}$ The identical size of $30 \times 30 \mathrm{~mm}^{2}$ was used for irradiation of all samples. Despite a small exit window of the microprobe, the large area was irradiated by sample moving. The dose of irradiation was varied by changing the current of the ion beam so as to be keeping the exposure time of $22 \mathrm{~min}$. The initial energy of $2 \mathrm{MeV}$ of protons and $7 \mathrm{MeV}$ of $\mathrm{He}^{2+}$ particle were used, but the cells were exposed at the energy 1.1 and $2.5 \mathrm{MeV}$, respectively, as the ions lost part of the energy within the microprobe nozzle and the shield of the biological sample holder. In gray unit, confluent cell monolayers were irradiated by the same doses $0.5,1,3,5$ Gy of protons and $\mathrm{He}^{2+}$ ions. The biological response of fibroblasts to acute irradiation was assessed using clonogenic and micronuclei (MN) formation assays.

Colony formation analysis as survival test was performed for 250 or 500 cells per dish growing during 14 days in standard conditions for cell cultivation. A photo of dishes with colonies obtained by seeding of 500 cells per dish irradiated by $0,0.5$, 1, 3, 5 Gy of $\mathrm{He}^{2+}$ particle beam can be seen on Fig. 13(a).

The results of micronuclei formation test data under $\mathrm{He}^{2+}$ and proton irradiation at the same dose range show that $\mathrm{He}^{2+}$ particles cause heavier DNA damage. For instance, $45 \%$ binuclear cells (BNCs) containing micronuclei $(\mathrm{MN})$ have been observed after irradiation by $1 \mathrm{~Gy}$ of $\mathrm{He}^{2+}$ particles in comparison with about $18 \% \mathrm{BNC}+\mathrm{MN}$ after proton irradiation [Fig. 13(b)]. Higher doses of $\mathrm{He}^{2+}$ irradiation induced also cell senescence which was not observed in proton-irradiated cells. Further increase in MN formation after 3-5 Gy $\mathrm{He}^{2+}$ ion-irradiation was stopped by the replicative senescence in $\mathrm{He}^{2+}$-irradiation treated cells but not in proton-irradiated.

The external beam application on the precise 2D elemental mapping in environmental studies, studies of biological samples, archaeological artefacts, and geological samples is planned in our laboratory. Typically, the target is encircled by an array of detectors; at least two X-ray detectors are normally used: a thin window detector for soft X-rays and a detector with a large solid angle but equipped with an additional absorber for hard X-rays. The external microbeam setup will be improved to be versatile and allow all IBA techniques to be used individually or in combination, namely, PIXE-PIGERBS with protons and PIXE-RBS with helium ions. The target region will be flushed with helium to reduce $\mathrm{X}$-ray absorption and X-ray background arising from interaction of the ion beam with Ar in air. 

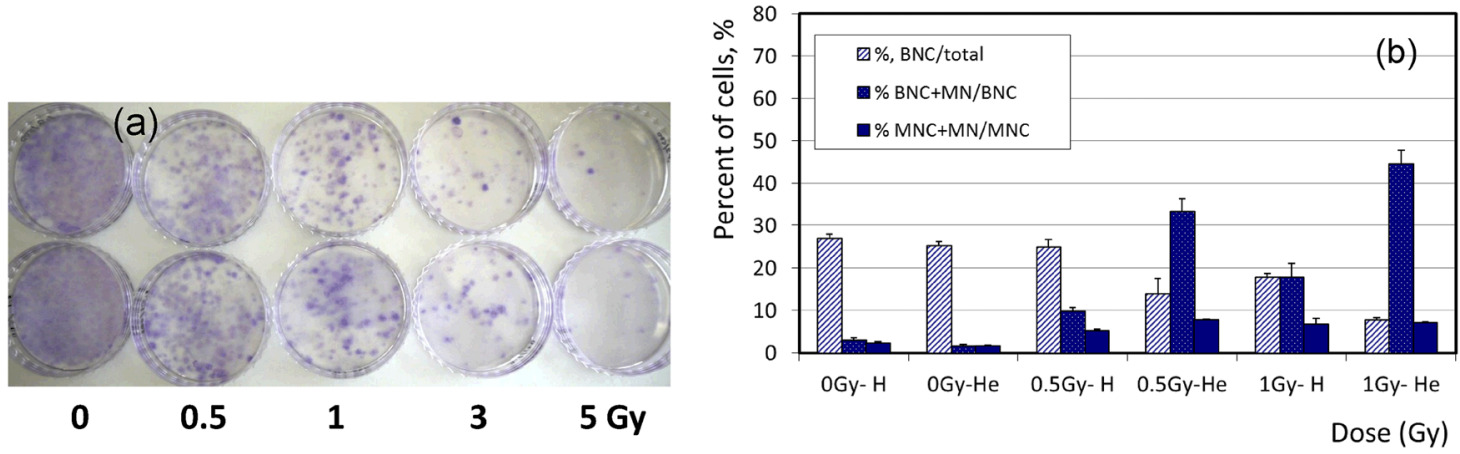

FIG. 13. Survival value of the biological cells under irradiation: (a) $\mathrm{He}^{2+}$ irradiation effect on colony formation of human fibroblasts. (b) Micronuclei formation in human neonatal fibroblasts after irradiation by different doses of protons and $\mathrm{He}^{2+}$ particles.

\section{CONCLUSIONS}

Over the last decade, the modern facilities for ion beam analysis of materials were developed at the NPI AS of the Czech Republic. One of them, scanning microbeam, was described in the present article in detail with our instrumental improvements performed mainly for ion beam micromachining. The resolution of $2 \times 3 \mu \mathrm{m}^{2}$ at $100 \mathrm{pA}$ of $2 \mathrm{MeV}$ proton beams is used for routine experiments of ion beam analysis of materials. The best resolution of $0.3 \times 0.5 \mu \mathrm{m}^{2}$ for carrying out the STIM measurement of materials is shown. The application of the microprobe for investigation of the chemical element distribution in a solid, creation, and determination of $3 \mathrm{D}$ structure in polymers and biological cell irradiation were considered. Our future steps in microprobe development consist of improving the resolution and electronics system. We plan to analyze benefits of a changing configuration of the triplet of magnetic quadrupole lenses as the focusing system (to consider a distributed triplet for our facility), decrease working distance and increase ion beam axial brightness. In order to expand our experiments with external ion beam, we will add detectors to the end-stage. This will allow not only irradiate samples but also perform analysis of such types of samples that cannot be placed into the chamber because of large size or vacuum environment.

\section{ACKNOWLEDGMENTS}

The instrumental development has been carried out at the CANAM (Centre of Accelerators and Nuclear Analytical Methods) infrastructure LM 2015056. This publication was supported by OP RDE, MEYS, Czech Republic under the project CANAM OP, CZ.02.1.01/0.0/0.0/16_013/0001812. The scientific results were obtained with the support of the GACR Project No. P108/12/G108.

\section{REFERENCES}

${ }^{1}$ R. Hinrichs, A. P. L. Bertol, and M. A. Z. Vasconcellos, Nucl. Instrum. Methods Phys. Res., Sect. B 363, 75 (2015).

${ }^{2}$ A. D. C. Alves, S. Thompson, C. Yang, and D. N. Jamieson, Nucl. Instrum. Methods Phys. Res., Sect. B 269, 2355 (2011).
${ }^{3}$ A. Macková, V. Havránek, J. Vacík, L. Salavcová, and J. Špirková, Nucl. Instrum. Methods Phys. Res., Sect. B 249, 856 (2006).

${ }^{4}$ R. Huszank, A. Simon, E. Szilágyi, K. Keresztessy, and I. Kovács, Nucl. Instrum. Methods Phys. Res., Sect. B 267, 2132 (2009).

${ }^{5}$ W. Maenhaut, Nucl. Instrum. Methods Phys. Res., Sect. B 363, 86 (2015).

${ }^{6}$ A. Macková, L. Salavcová, J. Špirková, R. Groetzschel, and F. Eichhorn, Nucl. Instrum. Methods Phys. Res., Sect. B 249, 339 (2006).

${ }^{7}$ R. Ishigami, Y. Ito, and K. Yasuda, Nucl. Instrum. Methods Phys. Res., Sect. B 266, 1319 (2008).

${ }^{8}$ A. Mackova, P. Malinsky, B. Svecova, P. Nekvindova, and R. Grötzschel, Nucl. Instrum. Methods Phys. Res., Sect. B 268, 2042 (2010).

${ }^{9}$ R. Minqin, J. A. van Kan, A. A. Bettiol, L. Daina, C. Y. Gek, B. B. Huat, H. J. Whitlow, T. Osipowicz, and F. Watt, Nucl. Instrum. Methods Phys. Res., Sect. B 260, 124 (2007)

${ }^{10}$ P. Malinský, A. Macková, R. Mikšová, H. Kováčiková, M. Cutroneo, J. Luxa, D. Bouša, B. Štrochová, and Z. Sofer, Phys. Chem. Chem. Phys. 19, 10282 (2017).

${ }^{11}$ X. Liu, Y. Miao, M. Li, M. K. Kirk, S. A. Maloy, and J. F. Stubbins, J. Nucl. Mater. 490, 305 (2017).

${ }^{12}$ R. Mikšová, A. Macková, H. Pupikova, P. Malinský, P. Slepička, and V. Švorčík, Nucl. Instrum. Methods Phys. Res., Sect. B 406, 199 (2017).

${ }^{13}$ G. Gawlik, P. Ciepielewski, J. Jagielski, and J. Baranowski, Nucl. Instrum. Methods Phys. Res., Sect. B 406, 683 (2017)

${ }^{14}$ V. Hnatowicz, V. Havránek, J. Bočan, A. Macková, J. Vacík, and V. Švorčík, Nucl. Instrum. Methods Phys. Res., Sect. B 266, 283 (2008).

${ }^{15}$ Y. Q. Li, C. Habchi, X. Liu, Y. Y. Liu, Y. Zheng, X. Y. Li, and H. Shen, Fusion Eng. Des. 88, 188 (2013).

${ }^{16}$ N. Guo, H. Y. Lu, Q. Wang, J. Meng, D. Z. Gao, Y. J. Zhang, X. X. Liang, W. Zhang, J. Li, X. J. Ma, and H. Shen, Nucl. Instrum. Methods Phys. Res., Sect. B 404, 162 (2017).

${ }^{17}$ R. Sano, S. Hayakawa, H. Hayashi, Y. Ishii, and H. Nishikawa, Nucl. Instrum. Methods Phys. Res., Sect. B 404, 228 (2017).

${ }^{18}$ I. Bányász, I. Rajta, G. U. L. Nagy, Z. Zolnai, V. Havranek, S. Pelli, M. Veres, S. Berneschi, G. Nunzi-Conti, and G. C. Righini, Nucl. Instrum. Methods Phys. Res., Sect. B 331, 157 (2014).

${ }^{19}$ Y. Yao, C. Zhang, S. K. Vanga, A. A. Bettiol, and F. Chen, Opt. Mater. 35, 2257 (2013).

${ }^{20} \mathrm{~T}$. Calligaro, J. C. Dran, E. Ioannidou, B. Moignard, L. Pichon, and J. Salomon, Nucl. Instrum. Methods Phys. Res., Sect. B 161, 328 (2000).

${ }^{21}$ L. Giuntini, M. Massi, and S. Calusi, Nucl. Instrum. Methods Phys. Res. Sect. A 576, 266 (2007)

${ }^{22}$ L. Breuer, F. Meinerzhagen, M. Bender, D. Severin, and A. Wucher, Nucl. Instrum. Methods Phys. Res., Sect. B 365, 482 (2015).

${ }^{23}$ B. N. Jones, V. Palitsin, and R. Webb, Nucl. Instrum. Methods Phys. Res., Sect. B 268, 1714 (2010). 
${ }^{24}$ See http:/ / www.ujf.cas.cz/en/research-development/open-access / for information about access to the institute facilities..

${ }^{25}$ G. Devès, S. Matsuyama, Y. Barbotteau, K. Ishii, and R. Ortega, Rev. Sci. Instrum. 77, 056102 (2006).

${ }^{26}$ G. W. Grime and M. Dawson, Nucl. Instrum. Methods Phys. Res., Sect. B 104, 107 (1995).

${ }^{27}$ A. G. Ponomarev, K. I. Melnik, and V. I. Miroshnichenko, Nucl. Instrum. Methods Phys. Res., Sect. B 231, 86 (2005).

${ }^{28}$ A. Dymnikov and R. Hellborg, Nucl. Instrum. Methods Phys. Res., Sect. A 330, 323 (1993).

${ }^{29}$ P. Malinský, M. Cutroneo, A. Macková, V. Hnatowicz, M. Florianová, M. Boháčová, D. Bouša, and Z. Sofer, Surf. Coat. Technol. 342, 220 (2018).
${ }^{30}$ P. Malinský, M. Cutroneo, A. Macková, V. Hnatowicz, K. Szökölová, M. Bohačová, J. Luxa, and Z. Sofer, Surf. Interface Anal. 50, 1110 (2018).

${ }^{31}$ G. U. L. Nagy, V. Lavrentiev, I. Bányász, S. Z. Szilasi, V. Havranek, V. Vosecek, R. Huszánk, and I. Rajta, Thin Solid Films 636, 634 (2017).

${ }^{32} \mathrm{M}$. Cutroneo, V. Havranek, A. Mackova, V. Semian, L. Torrisi, and L. Calcagno, Nucl. Instrum. Methods Phys. Res., Sect. B 371, 344 (2016).

${ }^{33}$ M. D. A. Michaelidesová, J. Vachelová, J. Konířová, I. Falková, M. Falk, V. Havránek, J. Štursa, and V. Zach, in Book of Abstracts Conference on Cell Biology and Radiobiology, Brno, Czech Republic, 8-11 September 2016, edited by M. Falk (IBP CAS, v.v.i., Brno, 2016), p. 13. 